\title{
Las misiones de divulgación cultural en la experiencia pedagógica de Olga y Leticia Cossettini (1) (Rosario, 1935-1950)
}

\author{
María del Carmen Fernández \\ María Elisa Welti \\ (Escuela de Ciencias de la Educación) \\ (Universidad Nacional de Rosario. Argentina) \\ María Eugenia Guida \\ (Escuela de Ciencias de la Educación) \\ (Universidad Nacional de Rosario. CONICET. Argentina)
}

\begin{abstract}
Resumen
El trabajo se centra en un aspecto de la propuesta educativa desarrollada en la ciudad de Rosario por Olga y Leticia Cossettini durante el período 1935-1950: las Misiones de Divulgación Cultural. Estas Misiones tenían como finalidad la difusión de la ciencia y el arte en la comunidad. Para ello se desplazaban maestros y alumnos a distintos barrios e instituciones de Rosario y en algunas oportunidades su difusión trascendió la ciudad y la provincia de Santa Fe. En trabajos anteriores el equipo de investigación se abocó al estudio de diversos aspectos de la Escuela Serena (nombre que recibió la experiencia desarrollada por las Cossettini durante el período mencionado en la Escuela Dr. Gabriel Carrasco de la ciudad de Rosario); en este caso indagamos acerca de las Misiones Culturales, aspecto escasamente explorado de la propuesta, puesto que consideramos que forman parte del conjunto de innovaciones pedagógicas llevadas a cabo en la Escuela Serena y además, un peculiar e interesante modo de concebir la relación entre escuela, cultura y sociedad.

Por otra parte, buscamos identificar aquellas experiencias similares en América Latina con las cuales las hermanas Cossettini establecieron alguna relación.
\end{abstract}

\section{Palabras clave}

Escuela Serena - Misiones Culturales - Comunidad - Escolanovismo - Pedagogía.

\section{Summary}

This article focuses on one aspect of the educational proposal developed in the city of Rosario by Olga Leticia Cossettini during the period 1935-1950: Cultural 
Outreach Missions. These missions were aimed at the dissemination of science and art in the community. To do so teachers and students moved to different districts and institutions of Rosario, and its dissemination sometimes transcended the city and the province of Santa Fe. In previous presentations, the research team undertook the study of various aspects of the Serena School (name which was developed by Cossettini's experience during the period mentioned in Dr. Gabriel Carrasco School, Rosario); in this particular case we inquire about the Cultural Missions, little explored aspect of the proposal, since we consider it part of a set of pedagogical innovations carried out in the Serena School, and they mean a peculiar and interesting way of conceiving the relationship between school, culture and society. Moreover, we seek to identify those similar experiences in Latin America with which the sisters Cossettini have established some relationship.

\section{Key Words:}

Serena School - Cultural Missions - Scholanovism - Community - Pedagogy.

\section{Introducción}

El análisis de la conformación del campo pedagógico durante la década del `30 muestra una compleja trama de posiciones educativas, políticas, ideológicas y estéticas. En esta trama encontramos posturas vinculadas al espiritualismo ("espiritualizar la escuela" fue el lema elegido por el Dr. Juan Terán quien fuera Presidente del Consejo Nacional de Educación a principios de la década), al nacionalismo y al catolicismo; vemos también en la época búsquedas tendientes a una "reforma moral" así como una superposición de valores y rituales católicos y espiritualistas laicos en el discurso escolar.

Por otro lado, en esos años se produce la difusión de las corrientes "escolanovistas" en el país; corrientes que se articulan de modo complejo con distintas fuerzas políticas como el radicalismo, el socialismo y la democracia progresista y que dan lugar al desarrollo de experiencias de escuela nueva de diverso alcance y tenor. Las reformas educativas que desde el estado se impulsan entonces no son ajenas al influjo de estas ideas en el ámbito local.

Al mismo tiempo se consolida en la Argentina el proceso de modernización iniciado en la década del '20; proceso que evidencia éxitos y transformaciones notables en el terreno del arte y de la cultura. El afianzamiento de los nuevos medios de comunicación (revistas, diarios, radio) constituyen una clara evidencia de ello.

Es en este contexto en el que tiene lugar la experiencia llevada adelante por las hermanas Olga (1898-1987) y Leticia Cossettini (1904-2004) 
en la Escuela "Dr. Gabriel Carrasco" de la ciudad de Rosario. La primera de ellas desde la dirección escolar y la reflexión teórica-didáctica y la segunda desde la docencia y la promoción de actividades artísticas, gestaron una de las más singulares experiencias de entre las que intentaron llevar a la práctica, en la Argentina, los principios de la Escuela Nueva. Se trató del proyecto de la Escuela Serena, que se inició en 1935 con el nombramiento de Olga como directora de la Escuela, ubicada en el barrio de Alberdi de la ciudad de Rosario, provincia de Santa Fe, y más precisamente con el otorgamiento a dicha escuela del carácter de experimental, al margen del régimen escolar vigente, a fines del mismo año (2). A ella asistían los hijos de los vecinos de Alberdi que conformaban un grupo social policlasista. En 1950 con la cesantía definitiva de Olga Cossettini, llegó a su fin la existencia fáctica de la experiencia de la Escuela Serena, pero comenzó otra existencia casi mítica (3).

Si bien son múltiples las aristas a indagar en esta experiencia, muchas de ellas ya analizadas en trabajos precedentes (4), nos centramos en este caso en la consideración de una de sus peculiares actividades: las misiones de divulgación cultural.

\section{La Escuela Serena: innovaciones y principios}

La escuela implementó un conjunto de propuestas innovadoras, algunas similares a otras propuestas escolanovistas, y otras propias y singulares, que contribuyeron a la trascendencia de la experiencia. El protagonismo de los alumnos fue una de las características que articuló el plan de trabajo institucional. La creación del Centro Estudiantil Cooperativo, la realización de tareas a cargo de los niños/as en la Biblioteca y el Laboratorio, la elaboración y puesta en marcha de las excursiones infantiles, la permanente preocupación por las producciones estéticas, la organización de comisiones de alumnos para la atención del jardín, huerta y animales, la edición de la revista "La voz de la Escuela", la creación del Teatro Infantil y Títeres, la formación del Coro de Pájaros, la implementación de los conciertos fotoeléctricos, las visitas de intelectuales como Juan Ramón Jiménez, Margarita Xirgu, Javier Villafañe, Gabriela Mistral, etc.

La implementación de estas innovaciones responde a un conjunto de ideas y concepciones acerca de la infancia, de la educación y de la función de la escuela que tanto Olga como Leticia Cosettini manifestaron en más de una oportunidad. Entendieron que en un proyecto educativo "Ios valores estéticos están íntimamente ligados a los valores morales, físicos e intelectuales" (5). Estos valores conducen a la consolidación de hábitos de belleza; y al mismo tiempo permiten, junto a la palabra escrita, la expresividad de 
los "estados de alma" peculiares e irrepetibles de cada uno de los niños y niñas, fundamento esencial de su "expresividad creadora".

Dicha "expresión creadora" infantil será precisamente el tema del libro El niño y su expresión que se publica en 1940. El libro incluye una conferencia pronunciada por Olga Cossettini y trabajos de los alumnos de la Escuela Experimental "Dr. Gabriel Carrasco": poemas, reproducciones de acuarelas, dibujos y trabajos prácticos de los alumnos. En dicha conferencia, Olga adscribe explícitamente a la tesis romántica del espontaneísmo creativo del niño, espontaneísmo que cuenta con un potencial afectivo, y que se expresa mediante una actividad del espíritu y del cuerpo.

Es por ello que la escuela debe actuar en sentido estrictamente contrario a la manera en que lo hiciere la escuela tradicional, y transformarse, por ende, en un espacio ideal que posibilite, incentive y concrete la "expresión creadora": "cuando el medio didáctico ejercita la actividad imaginativa ayudándola simplemente a crecer y a manifestarse, sabemos de cuánto es capaz el alma del niño, abierta a la emoción y a la belleza del mundo. Los claros dibujos que ilustran sus trabajos, sus canciones, la danza y el juego, la música con que alegran sus paseos, los libros serenos, poesía fresca, ciencia amena, dan a la infancia savia de crecimiento, sin turbarla, sin ajarla, ayudándola a crecer" (6).

El arte, la ciencia, la solidaridad social conforman una trama de la cual la escuela no puede permanecer ajena. "La escuela, en general, cuenta con dos medios donde los valores se hacen realizables: lo que llamamos contorno natural, barrio, lugar, paraje, donde es posible verificar las primeras valoraciones científicas y estéticas; y el mundo o medio social donde se realizan las formas de la conducta o valores éticos. La escuela, está obligada a contar, fundamentalmente, con estos dos medios para cumplir con su finalidad educadora" (7).

\section{Las Misiones Infantiles de Divulgación Cultural en la Escuela Serena: una primera aproximación}

La Escuela Serena promovió que los alumnos realizaran sus actividades escolares, no en forma aislada sino conformando grupos de trabajo, cooperando y estableciendo vínculos de solidaridad y fraternidad. Este propósito cobra envergadura cuando traspasa los límites materiales de la escuela, cuando los distintos saberes y lenguajes enseñados y aprendidos, comienzan a ser difundidos, dando cuenta de una actitud de compromiso social, en la plaza, en el barrio, en la ciudad, en las localidades vecinas. Señala Olga Cossettini que el programa de la Escuela para los niños y niñas entre 12 y 14 años "ejercita la conducta como miembro ya integrante 
de la sociedad, aportando su ayuda en la práctica de la asistencia social, llevando al seno de la sociedad, por intermedio de las Misiones Infantiles de Divulgación Cultural, su teatro, sus experiencias científicas, su creación, que se traduce en poemas, teatro, dibujo, plástica y construcciones" (8).

Estas Misiones comienzan a realizarse a fines de 1936 y se extienden, según los registros, hasta el año 1941. Se llevaban a cabo durante el mes de noviembre y de ellas participaban niños y niñas entre 10 y 14 años, con el objetivo de divulgar en el medio la experiencia adquirida en una comunidad más pequeña como la escuela y el aula.

Olga Cossettini define a las Misiones como "la escuela en la calle, en contacto con el pueblo, a quien lleva su saber y su experiencia. Expresión de vida de la escuela que sale de sus límites estrictos y busca contacto con el mundo que la rodea" (9). Olga, además, ve en las Misiones la posibilidad de lograr una "verdadera formación integral" (10).

Las Misiones consistían en una jornada de trabajo con los vecinos, donde los niños se convertían en los difusores de conocimiento y contaban con un amplio programa de cultura general, que abarcaba desde expresiones artísticas hasta temas vinculados a la ciencia, la biología y la prevención de enfermedades (11). Eran anunciadas con anticipación mediante carteles y programas confeccionados por los niños, quienes en la fecha señalada trasladaban todos los elementos necesarios para su concreción: mesas, vestuario, láminas, etc.

En los programas de las Misiones Culturales se evidencia la amplitud y diversidad temática de las mismas, de las cuales es representativo el programa de la Misión del 18 Noviembre de 1940, en la cual, a diferencia de los años anteriores (12), deciden agrupar los temas en una sola Misión, resultando una jornada donde se trataron los siguientes:

$1^{\circ}$ Evolución de los medios de transporte

$2^{\circ}$ Nuestro puerto y nuestra chacra

$3^{\circ}$ La pintura, desde los primitivos a los contemporáneos

$4^{\circ}$ Arte indígena americano

$5^{\circ}$ Cómo se organiza un camping

$6^{\circ}$ Teatro de títeres

La preparación de cada Misión requería un conjunto de actividades por parte de maestras y alumnos: recolección de información, sistematización de la misma, formatos de exposición, preparación de materiales, etc. Los niños se convertían en investigadores, artesanos, artistas, actores. Claro que previamente la escuela había puesto a disposición de los niños aquellos elementos necesarios para incursionar en las distintas ramas del saber: historia geografía, biología, dibujo, teatro, música, etc. 
Cabe destacar que estas actividades llevadas a cabo en cada una de las Misiones eran registradas por maestros/as y alumnos/as. En el primer caso, el relato de las maestras se basaba en fundamentar la selección de los contenidos a dar y las estrategias de presentación que utilizarían para ello. Enfatizan también la respuesta de los niños, señalando particularmente el costado emocional y afectivo que la realización de estas misiones provocaba.

Así cuenta una maestra:

"Fue llegando el público, padres de los niños y amigos, que nos acompañaban una vez más y extraños que llegaban atraídos por la novedad. Y a todos atendieron los niños en su maravilloso "dar" explicaron y dieron a conocer sus opiniones y conversaron con el público" (13).

En el caso de los cuadernos de los alumnos se destacan también sus impresiones personales:

"Yo cuando hablaba estaba segura de lo que decía, sabía que instruía a mucha gente" (14).

"Todos los años desde que vengo a esta escuela llena de sabiduría y comprensión los niños han hecho misiones culturales (...) así han conseguido despertar el interés de mucha gente por medio del amor y la lucha" (15).

\section{Escuela, cultura y comunidad}

En las palabras de Olga Cossettini se puede reconocer la relación que se procuraba establecer, a través de estas Misiones, entre la escuela, el niño y la comunidad:

"La experiencia que el niño adquiere en la labor cotidiana de que lo aprendido en el núcleo familiar que es la clase, puede ser llevado fuera de la escuela, ya que la escuela como expresión de vida realiza un servicio social en el medio ambiente donde actúa y con el que está vinculado, unido, con ese traer y darse permanentemente, influye para que el niño se sirva de esa experiencia y cumpla su función transmisora de una manera lógica y natural. El hecho de que se muestre desenvuelto, entusiasta y pronto a satisfacer al público heterogéneo que lo rodea cuando sale a las plazas y calles a improvisar su tienda o su tablado misionero, es una prueba evidente de que está cumpliendo con un servicio social no impuesto por la escuela, sino nacido de esa 
intima relación de escuela y sociedad del que es agente activo y elemento útil" (16).

En esta relación, el vínculo con la comunidad es considerado como recíproco, fluido y de intercambio. El énfasis está puesto en la salida del niño hacia la comunidad y forma parte de un programa más amplio sostenido por los valores de la solidaridad y la asistencia. Este "servicio a la comunidad" no sería, entonces, el resultado de una necesaria acción social, sino algo inherente al rol que se pretende que los niños cumplan en ambos ámbitos.

Afirma Olga:

"Es nuestro anhelo, por ejemplo, formar en el niño el sentimiento de solidaridad humana, base de una comprensión entre los hombres que sólo será posible cuando se conozcan, se comprendan y se amen, hermanados en un común sentimiento de lealtad, de generosa ayuda, de mutua correspondencia. Aspiramos a que el niño, sienta como una necesidad natural la de "dar", dar de su alegría, de su belleza, de su emoción" (17).

"Se destaca en este sentido un modo de relación con el contexto socio-cultural que enfatiza la "salida" a la comunidad, no sólo como estrategia para la recuperación del espacio público, sino también como sostén de un ideal de escuela con un potencial transmisor de saber y cultura cuyo "vehículo" es el niño. En palabras de Olga: "La escuela debe llevar al seno de las comunidades el impulso de su enorme vitalidad" (18).

\section{La presencia de otras experiencias en la propuesta de la Escuela Serena}

A fines de la década del '30 Olga Cossettini publica un artículo en la Revista de Pedagogía bajo el título Misiones Culturales donde relata las características de las Misiones de la Escuela Serena. En ese artículo menciona como antecedente otra experiencia, que sin duda conocía, dado que se lleva a cabo una década antes: "En América Latina hubo maestros que se interesaron y llevaron a la práctica con el nombre de Misiones Culturales, pudiendo citar el nombre de Sabas Olaizola, maestro uruguayo, que hizo el ensayo en su escuela decrolyana de Las Piedras" (19).

Ahora bien ¿qué característica tenía esta experiencia uruguaya y con qué aspectos se identifica con la implementada en la Escuela Serena? Para aproximarnos a ella es necesario mencionar el contexto en el que tiene lugar: 
a mediados de la década del '20 se inicia en Uruguay un movimiento a favor de la escuela nueva que sigue los principios de las acciones desarrolladas por Decroly en Bélgica. Por entonces, el Consejo de Enseñanza Primaria y Normal autorizó a los Directores de escuela a realizar experiencias metodológicas enmarcadas en las nuevas corrientes educativas (20). Así se fundan las escuelas en las que se intenta llevar a la práctica las ideas escolanovistas: en Las Piedras y Progreso en 1925 y en Malvín en 1927. La primera de ellas a cargo del pedagogo uruguayo Sabas Olaizola (21) a quien se refiriera Olga en su artículo.

Para Olaizola fue la independencia y autonomía técnica lo que favoreció la concreción de estas experiencias: "la medida legal fue fundamental para la organización de escuelas nuevas en el país, fue su separación del régimen inspectivo común, por resolución del Consejo de Enseñanza primero, en 1925, y por ley de la República en 1927; ampliada en 1928, y en 1929; y reglamentada en 1933" (22).

Olaizola propuso un plan que pretendía acercar al niño a las cosas, a la vida. El trabajo escolar se desarrollaba en pabellones con tres ambientes diferenciados: el laboratorio, el taller y el aula. El primero es aquél en el que el trabajo del niño ahonda en lo teórico, realizando experiencias y observaciones directas. El taller se especializa en la labor manual. El aula se destina al trabajo intelectual, más apartado de las realidades concretas. Así, la unidad escolar no es la clase sino el pabellón que es atendido por tres maestros que tienen cualidades diferentes: uno se destaca en lo teórico, otro en lo económico y otro en lo estético.

La escuela de Las Piedras se organizaba en cuatro pabellones a los que asistían los niños según su edad. El primero de ellos era el de los cazadores, que remitía a la primera etapa del desarrollo de la humanidad, en el que se agrupaban los niños más pequeños (de 4 y 5 años). Luego, para los niños de segundo año, se establecía el pabellón de agricultores, en el que se encontraba la huerta y la estación meteorológica. Para tercer y cuarto año se crea el pabellón de artesanos, que comprendía una granja y su correspondiente producción artesanal. Finalmente, para los niños de quinto y sexto año se crea el pabellón de investigadores, en el que se abordaba la etapa del desarrollo científico y la madurez cívica del ciudadano.

Olaizola señala que una actividad de la escuela que contribuye a elevar el ambiente cultural educativo es la de las misiones infantiles. Estas consisten en un grupo de niños que se "traslada con su maestra a un sitio cualquiera de la ciudad donde puedan reunirse algunos vecinos: mujeres, niños, ancianos, etc. Previo anuncio colocado en ese lugar con unas horas de anticipación, los niños llegan al sitio elegido, y ante las personas que 
hayan querido asistir disertan, o leen sus trabajos, sobre los temas más adecuados" (23). Suelen ser, aclara Olaizola, temas de biología o meteorología; también los niños cantan o recitan.

En síntesis, para Olaizola "la misión infantil es pues un grupo de niños que con su maestro hace llegar al pueblo la cultura que recibe en la escuela (...) educación moral activa que se opera practicando el bien con los caudales del espíritu, al alcance del más humilde económicamente, todo un mundo de valores profundos se conciertan en este acto sencillo de organizar misiones infantiles como norma permanente de la enseñanza escolar" (24).

Pero además de esta experiencia, Olga Cossettini se vincula con otra también uruguaya. En este caso, el vínculo es más directo, dado que mantiene una relación epistolar (25) con su autor, Jesualdo Sosa (26). La propuesta escolanovista que Jesualdo lleva a cabo durante siete años (1928-1935) en la Escuela Rural № 56 de Canteras del Riachuelo en Colonia posee características singulares (27). En aquel momento Canteras del Riachuelo era poblado muy pequeño ubicado a $15 \mathrm{~km}$ de Colonia del Sacramento y cercano a la zona de areneras y canteras. Recién llegado a la institución Jesualdo describe las características del lugar: "El caserío miserable se ahoga entre la cal blanca de las casillas con sombreritos rojos y el parduzco de los ranchos semi caídos. De los ranchos que se inclinan como para acostarse y permanecen así par el resto de sus días Casillas de zinc y madera. Ranchos deshechos, de barro y paja de laguna. Y estamos rodeados de granito, de arena, de agua" (28).

Jesualdo elabora un programa de ensayo para implementar en el tercer año, que es autorizado por miembros del Consejo de Enseñanza Primaria y por la Directora de la escuela, y que se denominó Programa de Extensión Cultural. En él se articulaba la necesidad de dar respuestas a las carencias educativas del medio rural y la importancia otorgada a la expresión creadora. Si bien en un primer momento estuvo destinado a los estudiantes de tercer año, luego la escuela completa adhirió a la propuesta.

Debido a la característica de ensayo que asumió el programa, no contó con una estrategia metodológica previa, sino que la misma se fue configurando a medida que se avanzaba en el trabajo con los alumnos y la comunidad. La única premisa que, desde el inicio, se planteó fue la necesidad de permitir y desarrollar "la expresión creadora de los niños". Para lograr este objetivo Jesualdo pone en marcha dos actividades que organizan la tarea escolar: los centros de interés y el curso de extensión cultural o "paseos escolares". Así como la justificación de los primeros se basaba en la necesidad de que fuesen los alumnos quienes decidieran acerca de los 
temas a estudiar e investigar, en el caso de las actividades enmarcadas en la extensión cultural se fundamentaba en la importancia que Jesualdo le otorgaba a la estrecha relación entre la escuela y la comunidad.

En el curso de Extensión Cultural no sólo se exploraba el entorno natural y social (a través de visitas y campamentos) sino que la propia escuela se convirtió en un centro cultural local, en tanto se realizan en ella representaciones teatrales, festivales, etc.

Por otra parte, resulta interesante la creación por parte de la escuela de un periódico de aparición semanal (29) llamado "El Marrón" (aludiendo al color marrón de las canteras) que constaba de distintas secciones e informaba acerca de acontecimientos de la comunidad y también de las actividades realizadas por la escuela y fundamentalmente de las acciones de sus alumnos.

\section{Comentarios finales}

Según Savloff (30), en la relación escuela-comunidad se distinguen dos orientaciones fundamentales. Una es aquella en que la relación con lo comunitario o la comunidad se hace en función y aprovechamiento de la escuela misma, sea para su sostenimiento, sea para la enseñanza de los niños. La segunda orientación es de la escuela para la comunidad. Aquí la escuela se pone al servicio de la población en general en forma directa, no a través de los niños. Esto se realiza poniendo a disposición la escuela para actividades de tipo cultural, prestando servicios sociales, en materia de salud, de agricultura o con finalidades recreativas, cooperando con programas de desarrollo de la comunidad (Savloff entiende que esta modalidad se desarrolla raramente en Argentina "dado el carácter académico y enclaustrado de nuestra escuela común") y finalmente la escuela como centro de desarrollo de la comunidad, donde la primera toma la iniciativa de movilizar a la comunidad con objetivos de educación social y promoción del mejoramiento de la localidad o región.

Como bien señala el autor, cada uno de estos aspectos representa un modo distinto de la relación escuela-comunidad. Sin embargo cabe destacar que el autor al finalizar esta clasificación destaca enfáticamente a la experiencia Cossettini: "en cuanto a proyección social de la escuela, como el mejor modelo que podemos aplicar. Han hecho simplemente, escuela del pueblo. Ningún progreso hemos de proponer que no tenga en ellos el punto de partida y el espíritu orientador" (31).

Por otra parte, este dar supone una retribución, un retorno. Según Olga Cossettini a partir de este intercambio con la sociedad la escuela recibía los beneficios de los "amigos de la escuela". Se trata de personalidades 
de la cultura -artistas, directores de museo, intelectuales de la ciudad de Rosario, pero también de reconocimiento nacional e internacional- y/o de Asociaciones $u$ otros organismos, que atentos a las necesidades de la escuela, conforman un grupo de colaboración de diversa índole: desde el auspicio para la muestra de arte que se realizara en Estados Unidos a la instalación de un comedor escolar y un consultorio odontológico.

Se constituye entonces en esta experiencia una escuela viva, una escuela que se vincula con la comunidad sin descentrarse de su tarea de cultura y educación y que desarrolla de este modo lazos sólidos con el medio, sus actores y sus instituciones que le permiten ampliar sus fronteras constantemente.

\section{Notas Bibliográficas}

(1) Este artículo se enmarca en el Proyecto de Investigación "Las Misiones Culturales en la Escuela Serena (Rosario, 1935-1950)" radicado en el Instituto de Investigaciones de la Facultad de Humanidades y Artes de la Universidad Nacional de Rosario. Se parte de la hipótesis de que el estudio en profundidad de las Misiones Culturales puede contribuir a una comprensión precisa de la experiencia pedagógica/cultural que tuvo como centro de irradiación la actividad de las hermanas Cossettini. Entendemos que las Misiones Culturales constituyen un dispositivo pedagógico conformado por una trama de concepciones específicas sobre ciertas instituciones, ciertas prácticas, ciertos valores -educación, escuela, actividad, saberes, transmisión, comunidad, estrategias de difusión populares y masivas, ideología, posicionamientos políticos y sociales-, y una red de relaciones que se postulan entre ellos. Como entendemos que el estudio de la complejidad y riqueza de las Misiones sólo puede lograrse si se ponen en correlación con otros factores, algunos de los objetivos del Proyecto remiten al análisis de: el marco contextual del entramado político, cultural, pedagógico e institucional en el cual se llevaron a cabo; las propuestas educativas innovadoras vinculadas tanto con el movimiento escolanovista como con el progresismo americano que tuvieron lugar en la trama pedagógica argentina y la concreción de distintas experiencias que tomaron el formato y la denominación de "misiones" y su influencia en la propuesta de la Escuela Serena. La estrategia metodológica consiste en: una revisión sistemática y crítica de la bibliografía tanto sobre el proceso histórico, social y cultural argentino del período estudiado, como de las experiencias pedagógicas/culturales similares de la primera mitad del siglo XX; el relevamiento de archivos, fundamentalmente en Archivo Cossettini (IRICE); la compilación, clasificación y evaluación de fuentes documentales de diverso orden; el análisis de los documentos y entrevistas desde una matriz interpretativa socio-histórica. 
(2) Este otorgamiento del carácter de "escuela experimental" debe ser ponderado en el marco de una búsqueda de reformas en la enseñanza pública cristalizada en la sanción de la ley provincial № 2364 de "Educación Común, Normal y Especial" en 1934, bajo el gobierno del Dr. Luciano Molinas (1932-35), que proponía una enseñanza "conforme a los métodos activos", que "contemple las actividades recreativas y estéticas, juegos, deportes, cantos, música y declamación".

(3) Ponencia "Entre la pedagogía y la cultura: Olga y Leticia Cossettini, docentes e intelectuales" (Rosario, 1935-1950) autores: Mg. María del Carmen Fernández, Mg. María Elisa Welti, Prof. Eugenia Guida. Presentada en las IX Jornadas Nacionales de Historia de las Mujeres y IV Congreso Iberoamericano de Estudios de Género "Los caminos de la libertad y la igualdad en la diversidad". Rosario, 24 de Agosto de 2008.

(4) Biselli, R., Fernández, M. C. y Welti, M. E. (2005) "Imagen y enseñanza en la Escuela Serena (Rosario, 1935-1950)", en Anuario de la Sociedad Argentina de Historia de la Educación, № 6, Buenos Aires, Prometeo.

(5) Cossettini, O. (1935) Sobre un ensayo de Escuela Serena en la provincia de Santa Fe, en Olga Cossettini y Leticia Cossettini, Obras Completas, AMSAFE, Santa Fe, 2001, p. 66.

(6) Ibídem, p.198.

(7) Cossettini, O. (1945) La Escuela viva. Buenos Aires: Losada, p. 19.

(8) Ibídem, p. 24.

(9) Cossettini, O. La Escuela viva. Op. Cit., p. 100.

(10) En este aspecto resulta interesante la referencia a Dewey que Olga realiza, atendiendo a la idea de educación integral del niño y considerándolo un futuro miembro de la sociedad.

(11) A modo de ejemplo citamos el Programa de las Misiones Culturales realizadas en 1936. Los temas presentados por distintos grupos de alumnos eran: La hormiga; El alcoholismo; La mosca; Teatro (Adaptación de la ópera de Hunperdink, Hansel y Gretel); Sericicultura; Higiene Bucal; Apicultura; Flora local; Teatro (selección de obras de García Lorca). Cfr.: Cossettini, O. La escuela viva. Op. Cit. pp. 124-125.

(12) Entre 1936 y 1939 se realizaban con temas aislados y a veces en lugares y fechas diferentes.

(13) "Misión Infantil de divulgación cultural realizada por los alumnos de 4to. Grado el 23 de Noviembre de 1940". Relato de maestra, Cuaderno de clase, s/f, 1940. Archivo IRICE, Rosario.

(14) María Elena Sala, 10 años. Cuaderno de clase, s/f, 1940. Archivo IRICE, Rosario.

(15) Libertad Marín, 12 años, Cuaderno de clase, 17 de Noviembre de 1950, folio 1. Archivo IRICE, Rosario.

(16) Cossettini, O. (1939) "Misiones Culturales" en Revista de Pedagogía, Director Lorenzo Luzuriaga, Año I, Nº3, Segunda Época, p. 161. 
(17) Cossettini, O. Op. Cit., p. 99.

(18) Cossettini, O. Op. Cit., p. 100.

(19) Cossettini, O. Misiones Culturales, en Revista de Pedagogía Año 1, № 3, Segunda época. Buenos Aires, Losada, agosto 1939, p. 160.

(20) Cfr. Breve análisis histórico de la educación en el Uruguay, República Oriental del Uruguay Administración Nacional de Educación Pública Consejo de Educación Primaria Documento para la discusión - Abril 2007.

(21) Olaizola fue un reconocido maestro uruguayo nacido en 1894 quien en 1915 inicia su carrera como director de una escuela de la localidad de Salto, más tarde de Colonia y finalmente en 1921 asume la dirección de la escuela de Las Piedras donde a partir de 1925 inicia la organización de una escuela experimental. En 1927 visita la escuela de Decroly en Bélgica. Más tarde se desempeña como consultor de la OEA y como experto de la UNESCO. Fallece en Montevideo en 1974. Entre sus obras destacadas se encuentra "La Pedagogía Decroliana", publicada en 1927, "El método Decroly en el Plan de Las Piedras", de 1932, "La Escuela Nueva en el Uruguay" de 1935 y "El Plan de Maestros Asociados" de 1950.

(22) Olaizola, S. (1935) La escuela nueva en Uruguay. Montevideo, p. 90.

(23) Olaizola, S. Op. cit., 1935, pp. 147-148.

(24) Olaizola, S. Op. cit., 1935, p. 149.

(25) Las cartas escritas por Jesualdo a Olga Cossettini pertenecen a los años 1935 y 1945. Archivo IRICE, Rosario.

(26) En 1921 Jesualdo Sosa (1905-1982) obtiene una beca para estudiar magisterio en Montevideo. Ingresa al Instituto Normal para Varones ese mismo año y egresa en 1924. Al año siguiente es nombrado maestro interino en la escuela para varones $N^{\circ} 1$ de Rivera (localidad cercaba al pueblo donde había vivido durante su infancia) dado que era una práctica que el becado retornara, una vez egresado, a su lugar de origen antes de optar por otro destino. En 1926 gana, mediante un concurso de oposición, el cargo de maestro efectivo en la Escuela Experimental $\mathrm{N}^{\circ} 1$ de Malvín donde permanece durante dos años. En 1928, a los 23 años, contrae matrimonio con María Cristina Zerpa, Directora de la Escuela Rural $N^{\circ} 56$ de Canteras del Riachuelo en Colonia y ese mismo año solicita al Consejo de Enseñanza Primaria y Normal el traslado a esa escuela, traslado que le es otorgado.

(27) Jesualdo relata esta original experiencia educativa en su libro "Vida de un Maestro", publicado por primera vez en la ciudad de Buenos Aires por Editorial Claridad en 1935.

(28) Sosa, J. (1935) Vida de un Maestro, Buenos Aires, Editorial Claridad, p. 16.

(29) El periódico se publicó solamente durante el año 1930.

(30) Savloff, G. (1969) Educación de la Comunidad, Buenos Aires, Editorial Bibliográfica Argentina.

(31) Savloff, G. Idem anterior, p. 15. 


\section{Referencias Bibliográficas}

- Biselli, R., Fernández, M. C. y Welti, M. E. (2005) "Imagen y enseñanza en la Escuela Serena (Rosario, 1935-1950)" en Anuario de la Sociedad Argentina de Historia de la Educación $N^{\circ} 6$. Buenos Aires, Prometeo.

- Casullo, N. (2004) El tiempo de las vanguardias artísticas y políticas. Itinerarios de la Modernidad. Buenos Aires, Eudeba.

- Cossettini, O. (1945) La Escuela viva. Buenos Aires, Losada.

- Cossettini, O. (1935) "Sobre un ensayo de Escuela Serena en la provincia de Santa Fe", en Olga Cossettini y Leticia Cossettini (2001) Obras Completas. Santa Fe, Ediciones AMSAFE.

- Cossettini, O. (1939) "Misiones Culturales" en Revista de Pedagogía, Director Lorenzo Luzuriaga, Año I, № 3, Segunda Época. Buenos Aires, Losada.

- Olaizola, S. (1935) La escuela nueva en Uruguay. Montevideo.

- Pelanda, M. (1996) La escuela activa en Rosario: la experiencia de O. Cossettini. Rosario, IRICE.

- $\quad$ Puiggrós, A. (2003) El lugar del saber. Buenos Aires, Galerna.

- Puiggrós, A. (dir.) (1993) Escuela, Democracia y Orden (1916-1943). Buenos Aires, Galerna.

- Sarlo, B. (1988) Una modernidad periférica: Buenos Aires 1920 y 1930. Buenos Aires, Nueva Visión.

- Savloff, G. (1969) Educación de la Comunidad. Buenos Aires, Editorial Bibliográfica Argentina.

- Sosa, J. (1935) Vida de un Maestro. Buenos Aires, Claridad. 